\title{
Index Admission Laparoscopic Cholecystectomy, Our Experience after the Inception of Acute Care Surgery Programme
}

Muhammad Aakif ( $\triangle$ Aakif_khan2001@yahoo.com )

Cork University Hospital Group https://orcid.org/0000-0002-0506-9109

Zeeshan Razzaq

Cork University Hospital Group

James Byrne

Cork University Hospital Group

Hamid Mustafa

cork university hospital

Mudassar Majeed

Cork University Hospital Group

Ata Khan

Cork University Hospital Group

Mumtaz Bughio

Cork University Hospital Group

Fuad Aftab

Mallow General Hospital

Muhammad Amir Zaheer

Cork University Hospital Group

Akbar amin Achakzai

Bantry General Hospital

Hugo Jacobus Prins

Cork University Hospital Group

Mark Anthony Corrigan

Cork University Hospital Group

Henry Paul Redmond

Cork University Hospital Group

Mohammed yasser Kayyal

Cork University Hospital Group

Research article 
Keywords: Acute cholecystitis, index admission laparoscopic cholecystectomy, acute care surgery Posted Date: June 11th, 2020

DOI: https://doi.org/10.21203/rs.3.rs-32690/v1

License: (c) (i) This work is licensed under a Creative Commons Attribution 4.0 International License. Read Full License 


\section{Abstract}

Background: Gallstones are very common and frequently present as acute cholecystitis in up to $20 \%$ of patients with symptomatic disease, with wide variation in severity. Laparoscopic Cholecystectomy (LC) has become the gold standard for treatment of symptomatic disease. Although multiple studies have confirmed its safety, LC at index admission is still not widely practiced in Ireland. We present our experience of a cohort of patients who underwent index admission laparoscopic cholecystectomy at Cork University Hospital since the start of the acute care surgery program in May 2017.Aim: To determine the feasibility and safety of laparoscopic cholecystectomy at index admission.Methods: All adult patients who presented to an acute surgical assessment unit (ASAU) with symptomatic gall stone disease and underwent early laparoscopic cholecystectomy at index admission were included. The duration of this prospective cohort study was 27 months (May 2017 to July 2019). Patient demographics, indication for surgery, post-operative complications and conversion rates were recorded. In addition, timing of imaging, imaging findings and length of hospital stay were also noted.Results: A total of 233 patients underwent laparoscopic Cholecystectomy at index admission for various indications. Median age was 50 years with range between 16 - 88. Male to female ratio was 1:1.78. $142(61 \%)$ patients had acute cholecystitis, while the other indications were CBD obstruction (15.5\%), biliary colic $(11 \%)$ and acute biliary pancreatitis (10.5\%). 93 (40\%) patients had pre-op MRCP while 41 (17.6\%) underwent pre-op ERCP. All except 3 patients undergoing ERCP had pre-procedure MRCP. 2 patients had intra-operative cholangiograms. Overall morbidity was $4.7 \%$. In terms of complications, $3(1.3 \%)$ patients had bile leak and only $1(0.85 \%)$ had re-operation. There was 1 common bile duct injury and only 1 conversion to open surgery. There was no mortality in this case series. The average length of hospital stay was 5.6 days. (Range 2 to 14 days).Conclusions: Index admission laparoscopic cholecystectomy for acute cholecystitis, choledocholithiasis, biliary colic and acute biliary pancreatitis, has been a safe and feasible treatment option in our hospital. A safe practice can be ensured by adherence to this care pathway and a multidisciplinary, consultant-led service. Index cholecystectomy service can be provided safely to reduce disease-related morbidity and multiple re-admissions in patients awaiting interval surgery.

\section{Background:}

Gallstones are very common and in the western world approximately $10-15 \%$ of the adult population is affected by this disease. The third American National Health and Nutrition Examination Survey has reported that 6.3 million men and 14.2 million women aged between 20 to 74 in the United States have gallbladder disease. ${ }^{1-4}$ Gall stone disease is also a major health burden across Europe and cumulative incidence has been reported as $0.6 \%$ per year with an overall prevalence of $9 \%$ in females and $10 \%$ in males. ${ }^{5}$

Female gender, obesity, elevated non HDL cholesterol and gall bladder polyps have been identified as the main risk factors for gallstones. The majority of patients present with complications related to gall stones in the form of biliary colic, acute cholecystitis, acute pancreatitis and jaundice related to migration of gall 
stones into the common bile duct. Gallstones present as acute cholecystitis in up to $20 \%$ of patients with symptomatic disease, with wide variation in severity. ${ }^{6}$

Laparoscopic cholecystectomy (LC) has become the gold standard for treatment of symptomatic disease. There have been extensive studies on the management of acute cholecystitis in the emergency setting. More recently, Tokyo guidelines and World Society of Emergency Surgery (WSES) guidelines now

recommend early cholecystectomy during the index admission. ${ }^{7,8}$ Early cholecystectomy seems to have lower morbidity and mortality, lesser rate of conversion to open and shorter hospital stay if performed within 7-10 days of onset of symptoms. ${ }^{9}$

Still a major dilemma remains unsolved; that is the risk of major complications associated with this approach of early cholecystectomy, for example common bile duct injury. A relatively large population based study from Sweden showed that the risk of common bile duct injury is doubled for patients with acute cholecystitis in early cholecystectomy. ${ }^{10}$ Other studies have reported a wide range of conversion (4.9-20\%) from laparoscopic to open cholecystectomy despite the increase in surgical expertise during early cholecystectomy. ${ }^{11}$ However, most of these studies are under powered and hence there is no conclusive evidence to date with regards to increased risk of bile duct injury and conversion to open surgery in early cholecystectomy.

Based on this background, we planned to conduct a prospective cohort study at Cork University Hospital, Ireland after the inception of the acute care surgery programme in May 2017. The aim of the study was to assess the feasibility and safety of index admission laparoscopic cholecystectomy in our programme for various indications in symptomatic gall stone disease regardless of the timing of presentation and severity of the disease.

\section{Methods}

This was a prospective cohort study undertaken from May 2017 until July 2019 at Cork University Hospital Cork (CUH). CUH started its acute care surgery programme in May 2017 and a dedicated team consisting of experienced consultants and trainees were assigned to run the service. A separate pathway was devised for all patients presenting with symptomatic gallstone related issues. These patients were directly sent from the emergency department to the acute surgical assessment unit and then fast tracked for imaging, admission and then emergency surgery accordingly. We have the availability in our setup to get each relevant speciality on board early in managing complex patients with obstructive jaundice secondary to choledocholithiasis. In such patients, radiology (for imaging techniques including MRCP and interventions e.g. PTC) and gastroenterology (for ERCP) are involved early in their specialist roles.

All adult patients above the age of 16 years with proven gall stone disease on ultrasound and MRI, undergoing index admission early laparoscopic cholecystectomy have been included. Patients who did not undergo surgery because of personal preference to opt for interval delayed surgery have been excluded. 
Data was collected prospectively and recorded on excel sheets including patients demographics, presenting symptoms, imaging done preoperatively including findings of relevant imaging modalities i.e. ultrasound, CT, MRCP; and whether patients went through preoperative ERCP or not. In addition, we also recorded patient's date of admission and date of discharge and thus their total length of hospital stay.

We have documented complications following surgery including bile leak, common bile duct injury, haematoma formation and other related complications like post ERCP pancreatitis, bleeding, retained stones, re-admission rate and lower respiratory tract infections. We also documented if patients underwent post operative imaging of the biliary tree for any reason.

\section{Results}

A total of 233 patients underwent laparoscopic Cholecystectomy at index admission for various indications from May 2017 till July 2019. Median age for laparoscopic cholecystectomy was 50 years (Range 16-88 years). 148 patients were females (63.5\% of the cohort). The male to female ratio was

\section{1:1.78. (Pie chart 1)}

The majority of the patients $142(61 \%)$ had acute cholecystitis as the indication for index admission laparoscopic cholecystectomy. $15.5 \%$ presented with choledocholithiasis and underwent ERCP and their ducts were cleared before surgery. $11 \%$ patients presented with biliary colic. $10.5 \%$ had acute biliary pancreatitis. (Pie chart 2)

$93 \%$ of patients had pre-operative ultrasound (US) scans while $28.5 \%$ had a pre-operative CT scan as part of their work up. $40 \%$ of patients had pre-op MRCP out of which less than half (17.6\%) required an ERCP procedure. Only 5 patients required ERCP with CBD stent placement. 2 patients had intra-operative cholangiogram. (Table 1)

The overall morbidity was $4.7 \%$. In terms of complications, $3(1.3 \%)$ patients had a bile leak and $1(0.43 \%)$ had re-operation. 1 patient had a post-op hematoma which was drained percutaneously. 1 patient had their procedure abandoned because of bradycardia upon induction of anaesthesia. There was $1(0.43 \%)$ common bile duct injury, 1 conversion to open surgery and no 30 days mortality was reported. (Table 2 )

The average time between onset of symptoms and surgery was 5 days (Range 2 - 15). The average length of hospital stay varied from 3 days (range 1-7) for biliary colic patients to 9 days (range 4-14) for patients with choledocholithiasis. The overall average length of hospital stay was 5.6 days (Range: 2 - 14 days) (Table 3)

Patient's preoperative diagnosis and histology were matched and results analysed accordingly depending on imaging as in some cases normal gall bladder wall thickness was reported but was proved to be acute cholecystitis on final histology. We did not find any incidental gall bladder malignancies on final histology results. $1 \%$ patients had either polyps or adenomyomatosis on histology. 


\section{Discussion}

The timing of laparoscopic cholecystectomy (LC) i.e. either early or delayed as definite surgical management of acute cholecystitis has been controversial and a matter of debate for many years now. Most of the literature available to date has demonstrated that early LC has many benefits including shorter hospital stay and reduced morbidity. Delay of laparoscopic cholecystectomy after the diagnosis of biliary colic or acute cholecystitis may increase the probability of recurrent emergency admissions while awaiting elective delayed cholecystectomy. ${ }^{12}$ Clinical practice varies significantly worldwide and hence the observed differences regarding the timing of surgery have not yet been resolved. ${ }^{9}$

The latest Tokyo guidelines from 2018 have strongly suggested early LC within 72 hours of onset of symptoms as a safe and effective practice. ${ }^{7}$ Similarly, the World Society of Emergency surgery (WSES) in their 2016 guidelines have advised up to 10 days time period to be safe to operate in the context of early LC. Overall, the systematic review and meta-analysis of randomised controlled trials which the WSES used for these guidelines included clinical data from five of these six trials demonstrating no significant difference in the complication rate or conversion to open cholecystectomy between early and delayed laparoscopic cholecystectomy. Also, hospital stay was statistically shorter by 4 days in the early laparoscopic cholecystectomy group compared to the delayed laparoscopic cholecystectomy group. ${ }^{\mathbf{8}}$

In our study the average time between onset of symptoms and surgery was 5 days with a range from 215 days. In our experience, young male patients pose a significant risk towards complications because of their general trend of late presentation compared to females and also severity of the disease making it technically challenging to operate and thus increasing the risk of complications. In our cohort 3 patients had bile leak and all of them were males with average age of 42 years. Two of them had gangrenous gall bladders and necrotic cystic ducts and in one patient there was displacement of clips off the cystic duct. One patient was taken back to theatre for laparoscopic wash out and drainage. All three patients had post operative ERCP and stenting of their common bile ducts. Stents were removed 6-8 weeks later in our institute. One patient had common bile duct injury, which was identified per-operatively and a hepatobiliary surgeon's advice was taken and a drain was left in. Post operatively, patient underwent ERCP and stenting and common bile duct healed spontaneously with no further complications.

We have compared our results with a Cochrane review published a few years ago on early laparoscopic cholecystectomy and have found a significant difference in complications between studies included in that review and our findings. (Table 4) Our conversion from laparoscopy to open surgery was $0.43 \%$ as compared to $19-22 \%$ in the Cochrane review. Our overall morbidity was $4.7 \%$ which was significantly less than $26-28 \%$. In our opinion there is significant heterogeneity in the studies included in the Cochrane review. Many studies are relatively older and most set ups lack the dedicated emergency surgery services. 13

\section{Conclusion}


Index admission laparoscopic cholecystectomy for acute cholecystitis, choledocholithiasis, biliary colic and acute biliary pancreatitis, has been a safe and feasible treatment option in our hospital. It reduces the rate of morbidity associated with delayed surgery and patients' anxiety. A safe practice can be ensured by adherence to this care pathway and a multidisciplinary, consultant-led service. Index cholecystectomy service can be provided safely to reduce disease-related morbidity and multiple re-admissions in patients awaiting interval surgery.

\section{Abbreviations:}

LC(Laparoscopic cholecystectomy), ASAU (Acute surgical assessment unit ),ERCP ( Endoscopic retrogradecholangiopancreatogram),MRCP ( Magnetic resonance cholangiopancreatogram),CBD (common bile duct),WSES (world society of emergency surgery),PTC( percutaneous transhepatic cholangiogram),CT (computed tomogram), CUH (cork university hospital), USS (ultrasound scan)

\section{Declarations:}

Ethical Approval: Study approved by the research committe and all participants agreed to be part of research

Consent for publication: All participants agreed for publishing the research work

Availability of Supporting Data: All data is available for counter verification

\section{Competing Interests :None}

\section{Funding : None}

\section{Authors contributions: All authors did participate in the study at different levels}

MYK,MC came with main idea of the study, Data collection was done by MA and JB, Data was analysed by MA,ZR and HM. Initial manuscript was written by MA,MM and AUK, Manuscript was further edited by $Z R, M B, F A, M A Z, A A A$ and HJP, Final proof reading was done by HPR

\section{Acknowledgment}

We are very thankful to the pioneers of acute care surgery programme, radiology and gastroenterology departments for providing their service to our patients and being always there when needed.

\section{References:}

1. Gracie Ward. The natural history of silent gallstones: the innocent gallstone is not a myth. $\mathrm{N}$ Engl $\mathrm{J}$ Med. 1982;307::798-800. 
2. Portincasa P, Moschetta A, Palasciano G. Cholesterol gallstone disease. The Lancet. 2006;368(9531):230-9.

3. Kratzer W, Mason RA, Kächele V. Prevalence of gallstones in sonographic surveys worldwide. J. Clin. Ultrasound. 1999;27(1):1-7.

4. Pedersen G, Hoem D, Andrén-Sandberg $\AA$. Influence of laparoscopic cholecystectomy on the prevalence of operations for gallstones in Norway. Eur J Surg. 2002;168(8-9):464-9.

5. Shabanzadeh DM, Sorensen LT, Jorgensen T. Determinants for gallstone formation - A new cohort study and a systematic review with meta-analysis. Scand. J. Gastroenterol. 2016;51(10):1239-48.

6. Karanikas M, Bozali F, Vamvakerou V, Markou M, Memet Chasan ZT, Efraimidou E, et al. Biliary tract injuries after laparoscopic cholecystectomy-types, surgical intervention and timing. Ann. Transl. Med. 2016;4(9):163.

7. Yokoe M, Hata J, Takada T, Strasberg SM, Asbun HJ, Wakabayashi G, et al. Tokyo Guidelines 2018: diagnostic criteria and severity grading of acute cholecystitis (with videos). J. Hepatobiliary Pancreat. Sci. 2018;25(1):41-54.

8. Ansaloni et al. 2016. WSES guidelines on acute calculous cholecystitis. World Journal of Emergency Surgery (2016) 11:25

9. Cao AM, Eslick GD, Cox MR. Early laparoscopic cholecystectomy is superior to delayed acute cholecystitis: a meta-analysis of case-control studies. Surg. Endosc. 2016;30(3):1172-82.

10. Törnqvist B, Waage A, Zheng Z, Ye W, Nilsson M. Severity of acute cholecystitis and risk of iatrogenic bile duct injury during cholecystectomy, a population-based case-control study. World J. Surg. 2016;40(5):1060-7.

11. Amin A, Haider MI, Aamir IS, Khan MS, Khalid Choudry U, Amir M, et al. Preoperative and Operative Risk Factors for Conversion of Laparoscopic Cholecystectomy to Open Cholecystectomy in Pakistan. Cureus. 2019;11(8):e5446.

12. Salman B , Yüksel $O$, Irkörücü $O$ et al. Urgent Laparoscopic Cholecystectomy Is the Best management for biliary Colic. A Prospective Randomized Study of 75 Cases. Dig. Surg. 2005;22(1-2):95-9.

13. Gurusamy KS, Samraj K. Early versus delayed laparoscopic cholecystectomy for acute cholecystitis. Cochrane Database Syst Rev. 2006(4).

\section{Tables}

Table 1: Preoperative imaging 


\begin{tabular}{|lll|}
\hline Investigation & Number & Percentage\% \\
\hline US & 217 & 93 \\
\hline CT & 66 & 28.5 \\
\hline MRCP & 93 & 40 \\
\hline ERCP & 41 & 17.6 \\
\hline ERCP \& CBD Stent & 5 & 2.1 \\
\hline
\end{tabular}

Table 2: Complications

\begin{tabular}{|ll|}
\hline Biliary Complications & $\%(\mathrm{n}=)$ \\
\hline Bile Leak & $1.3 \%(\mathrm{n}=3)$ \\
\hline Haematoma & $1.3 \%(\mathrm{n}=3)$ \\
\hline Retained Stones & $0.86 \%(\mathrm{n}=2)$ \\
\hline CBD injury & $0.43 \%(\mathrm{n}=1)$ \\
\hline Conversion to Open & $0.43 \%(\mathrm{n}=1)$ \\
\hline Re-operation & $0.43 \%(\mathrm{n}=1)$ \\
\hline Total & $\mathbf{4 . 7 \% ( n = 1 1 )}$ \\
\hline
\end{tabular}

Table 3: Length of Stay.

\begin{tabular}{|lll|}
\hline Diagnosis & Average LOS (days) & Range (days) \\
\hline Acute cholecystitis & 5.5 & $2-14$ \\
\hline Choledocholithiasis & 9 & $4-14$ \\
\hline Acute Pancreatitis & 5 & $2-10$ \\
\hline Biliary Colic & 3 & $1-7$ \\
\hline Total & 5.6 & \\
\hline
\end{tabular}

Table 4 Comparison of our complications with the published data 


\begin{tabular}{|c|c|c|c|}
\hline Our Results & $\%(n=)$ & Cochrane Review EC & Cochrane Review LC \\
\hline Bile Leak & $1.3 \%(n=3)$ & $0.4 \%$ & $0.9 \%$ \\
\hline Haematoma & $1.3 \%(n=3)$ & \multirow[t]{3}{*}{$6.5 \%$} & \multirow[t]{3}{*}{$5 \%$} \\
\hline Retained Stones & $0.86 \%(n=2)$ & & \\
\hline CBD injury & $0.43 \%(n=1)$ & & \\
\hline Conversion to Open & $0.43 \%(n=1)$ & $19.7 \%$ & $22.1 \%$ \\
\hline Overall morbidity & $4.7 \%(n=11)$ & $26.6 \%$ & $28 \%$ \\
\hline
\end{tabular}

\section{Figures}

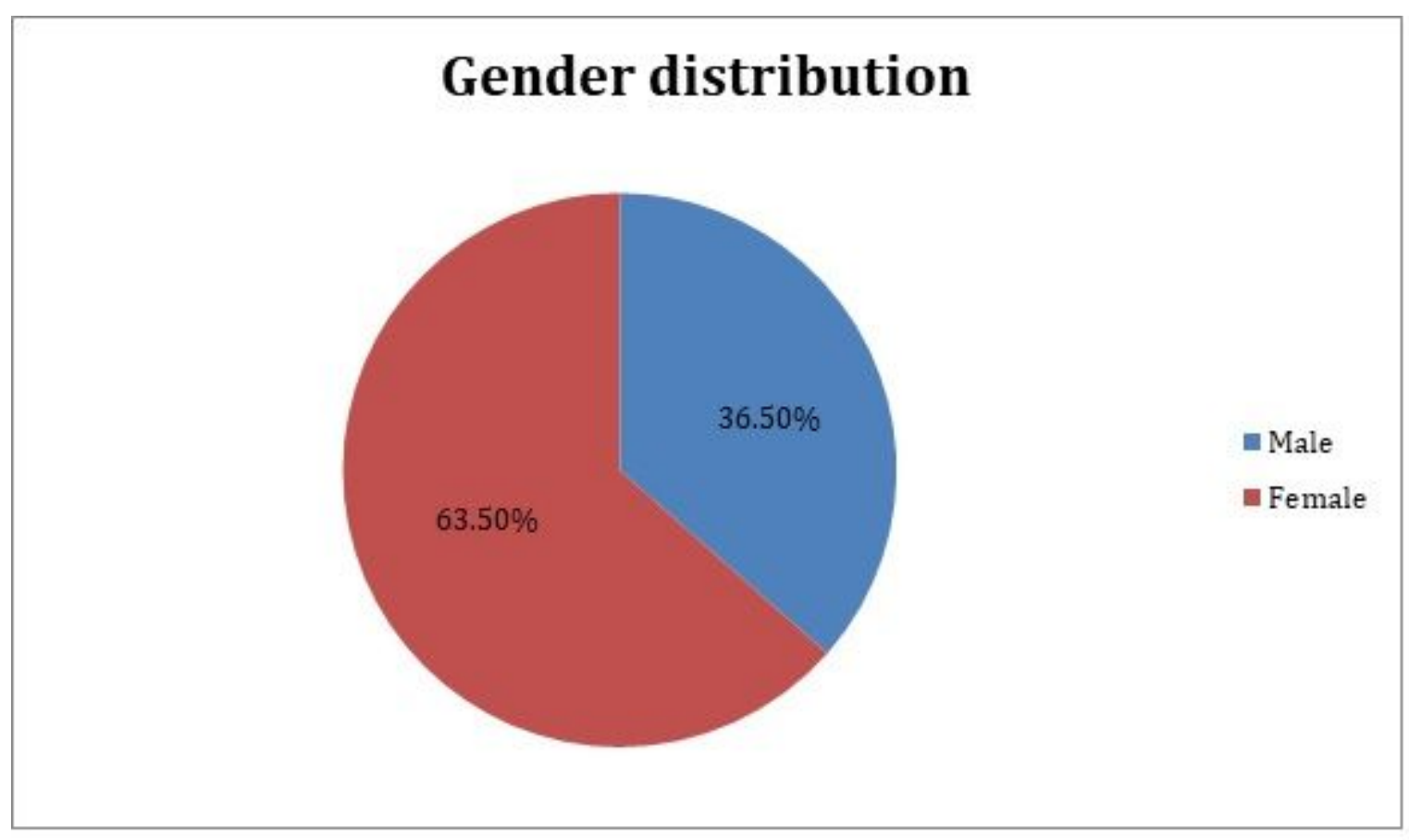

Figure 1

Pie chart 1 


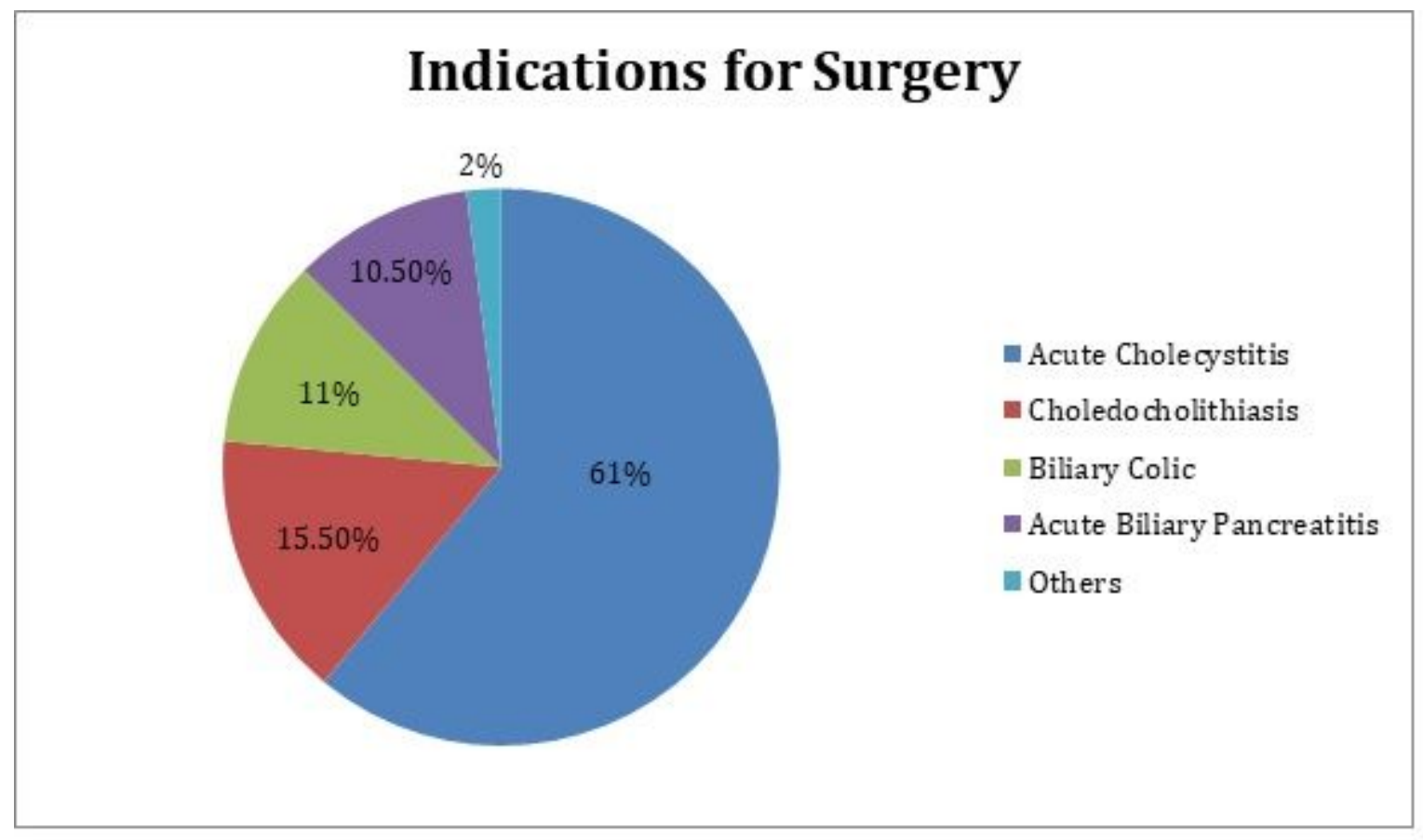

Figure 2

Pie chart 2 